\title{
Focused Ion Beam Etched Ring-Resonator in CVD-grown Ge-Sb-S Thin Films
}

\author{
C. C. Huang ${ }^{1}$, Y. -L. D. Ho ${ }^{2}$, K. Knight ${ }^{1}$, J. G. Rarity ${ }^{2}$, D.W. Hewak ${ }^{1}$ \\ ${ }^{1}$ Optoelectronics Research Centre, University of Southampton, Southampton, SO17 1BJ, United Kingdom \\ ${ }^{2}$ Department of Electrical and Electronic Engineering, University of Bristol, Bristol, BS8 1TR, United Kingdom \\ E-mail:cch@orc.soton.ac.uk
}

\begin{abstract}
Focused ion beam technique has been applied to fabricate ring resonators in $\mathrm{Ge}-\mathrm{Sb}-\mathrm{S}$ thin films for optoelectronic applications. The CVD-grown Ge-Sb-S thin films have been characterizatezed by micro-Raman, scanning electron microscopy, energy dispersive X-ray analysis and UV-VIS-NIR spectroscopy and the properties of the resonator are being assessed.
\end{abstract}

Keywords: CVD, Ge-Sb-S, thin films, FIB ring-resonator

\section{INTRODUCTION}

Thin amorphous chalcogenide films are very interesting materials because their diverse active properties allow their application as integrated planar optical circuits, as well as for memory and other optoelectronic applications [1-3]. In particular, germanium antimony sulphide (Ge-Sb-S) glasses have been intensively investigated because they can form stable ternary glasses within a large glass forming region and offer several properties of interest including high nonlinearity and photosensitivity [4]. The preparation of $\mathrm{Ge}-\mathrm{Sb}-\mathrm{S}$ thin films is usually performed by thermal evaporation techniques [5-7] with Ge-Sb-S target materials prepared by seal ampoule and melt-quench method. However, for the first time a direct fabrication method for $\mathrm{Ge}-\mathrm{Sb}-\mathrm{S}$ thin films with variable composition has been demonstrated. This new chemical vapour deposition (CVD) process has now been proven to yield high quality $\mathrm{Ge}-\mathrm{Sb}-\mathrm{S}$ thin films [8].

Focused ion beam (FIB) technique has been a very powerful tool since the late 1970s [9]. The FIB technique has been applied to fabricate of photonic crystal structures on the planar chalcogenide thin films [10]. In this paper, the FIB technique was used to pattern CVD-grown Ge-Sb-S thin film for optoelectronic applications.

\section{APPRATUS AND EXPERIMENTAL METHOD}

$\mathrm{Ge}-\mathrm{Sb}-\mathrm{S}$ thin films have been fabricated by the procedure, illustrated schematically in Fig. 1, which has provided a direct way to form chalcogenide thin film materials for a variety of optoelectronic applications. In this process, $\mathrm{Ge}-\mathrm{Sb}-\mathrm{S}$ amorphous thin films with variable compositions can be achieved over a temperature range from $120^{\circ} \mathrm{C}$ to $400^{\circ} \mathrm{C}$ from the following reactions:

$$
\begin{aligned}
& \mathrm{GeCl}_{4}+2 \mathrm{H}_{2} \mathrm{~S} \leftrightarrow \mathrm{GeS}_{2}+4 \mathrm{HCl} \\
& 2 \mathrm{SbCl}_{5}+3 \mathrm{H}_{2} \mathrm{~S} \leftrightarrow \mathrm{Sb}_{2} \mathrm{~S}_{3}+6 \mathrm{HCl}+2 \mathrm{Cl}_{2}
\end{aligned}
$$

The reactor used in the experiment was a horizontal quartz tube within a wire wound tube furnace capable of reaching $1200{ }^{\circ} \mathrm{C}$. The reactive gas, $\mathrm{H}_{2} \mathrm{~S}$, and the carrier gas, argon, for $\mathrm{GeCl}_{4}$, and $\mathrm{SbCl}_{5}$ were delivered through the mass flow controllers which provide $\pm 1 \mathrm{ml} / \mathrm{min}$ accuracy at the flow rate in the range of $50 \mathrm{ml} / \mathrm{min}-150 \mathrm{ml} / \mathrm{min}$.

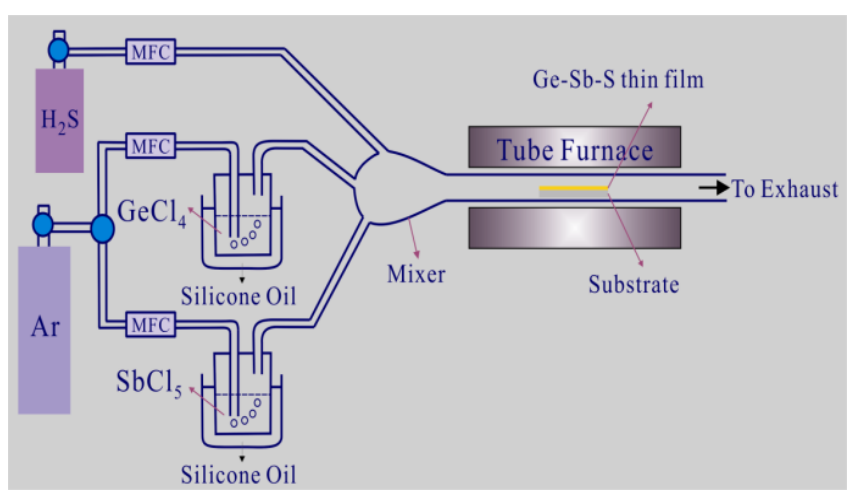

Fig. 1 Schematic of system used for Ge-Sb-S thin film deposition

\section{RESULTS AND DISCUSSION}

Ge-Sb-S thin films with different compositions were deposited on Schott N-PSK-58 glass slides and then characterized with a scanning electron microscope (SEM). As shown in Fig. 2, high quality Ge-Sb-S films have been successfully fabricated. The composition of these films was determined by energy dispersive X-ray (EDX) analysis and these results are also shown in Fig. 2. From the EDX results, the CVD process was calibrated for the deposition of Ge-Sb-S thin films with tuneable compositions. The thicknesses of these Ge-Sb-S films were also determined by SEM micrographs (Fig. 2) of the edge profiles of cleaved samples.

To confirm compositional changes and material phase, a Raman analysis was carried out with a RENISHAW Ramascope equipped with a CCD camera. A $633 \mathrm{~nm}$ He-Ne laser was used to excite the sample and the Raman shift spectrum was measured from $600 \mathrm{~cm}^{-1}$ to $100 \mathrm{~cm}^{-1}$ with a resolution of $1 \mathrm{~cm}^{-1}$. These Raman spectra, shown in Fig. 2 agrees with previous studies [8] although the intensities in Raman shift spectra of Ge-Sb-S thin films were different due to the thicknesses and refractive indices of the samples and the experimental conditions. The main Raman shift peak of these Ge-Sb-S films were found between $330 \mathrm{~cm}^{-1}$ and $300 \mathrm{~cm}^{-1}$ that was influenced by the ratio of $\mathrm{GeS}_{2}$ tetrahedral unit at $342 \mathrm{~cm}^{-1}$ and $\mathrm{Sb}_{2} \mathrm{~S}_{3}$ pyramidal unit at about $300 \mathrm{~cm}^{-1}$. From these Raman spectra, amorphous phases of the $\mathrm{Ge}-\mathrm{Sb}-\mathrm{S}$ thin films have been demonstrated.

Refractive indices $(n)$ and thicknesses of Ge-Sb-S thin films were measured by the method suggested by Swanepoel [11]. The transmission spectra of Ge-Sb-S 


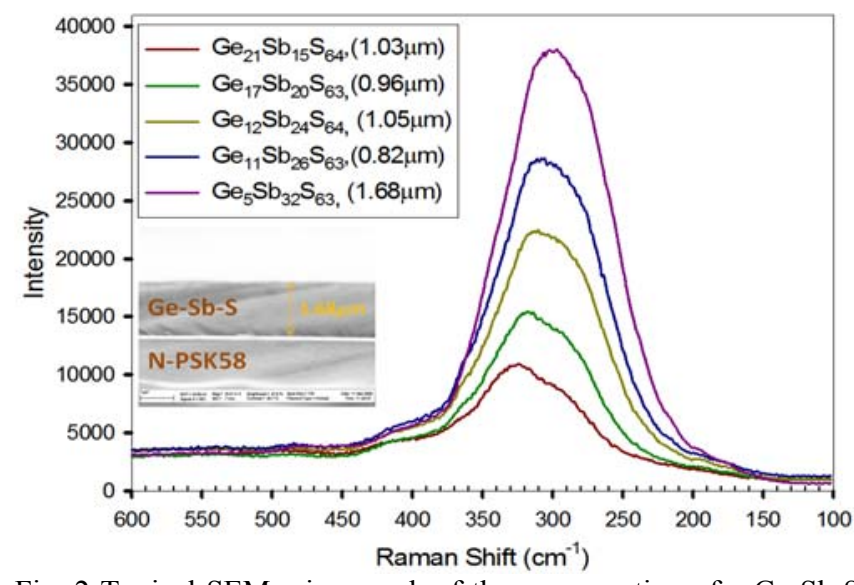

Fig. 2 Typical SEM micrograph of the cross section of a Ge-Sb-S thin film deposited on a Schott N-PSK58 substrate (inset) and Raman spectra of a series of Ge-Sb-S thin films.

amorphous thin films were measured by a Varian Cary 500 Scan UV-VIS-NIR Spectrophotometer at the resolution of 1 nm (Fig. 3). By using Swanepoel's method, we can calculate the refractive indices and thicknesses (Fig. 2) of the $\mathrm{Ge}-\mathrm{Sb}-\mathrm{S}$ thin films as a function of wavelength shown in Fig. 3. The refractive indices of $\mathrm{Ge}-\mathrm{Sb}-\mathrm{S}$ thin films increased with $\mathrm{Sb}$ content at all wavelengths for these $\mathrm{Ge}-\mathrm{Sb}-\mathrm{S}$ films. In addition, the refractive indices of these $\mathrm{Ge}-\mathrm{Sb}-\mathrm{S}$ films as a function of wavelengths can be fitted with the following Cauchy dispersion equation (Eq. 1).

$$
\left(n=n_{0}+A / \lambda^{2}+B / \lambda^{4}+C / \lambda^{6}\right)
$$

The thickness of each Ge-Sb-S film, measured by the single transmission method, agrees with the SEM measurements.

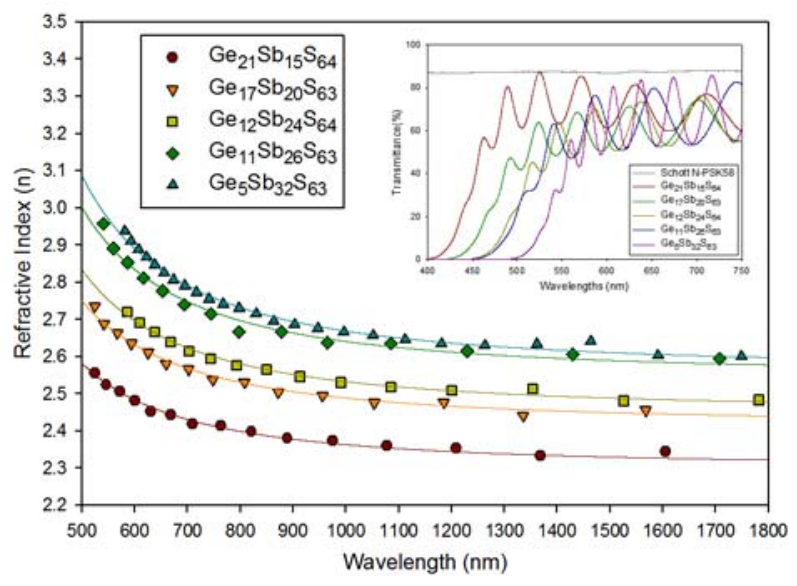

Fig

.3 Transmission spectra of Ge-Sb-S thin films deposited on Schott N-PSK58 substrates and refractive indices of Ge-Sb-S thin films with Cauchy dispersion fitting curves.

FIB has become a very attractive tool for making high-quality or high-precision micro- and nanostructures. In our experiments, a Ge-Sb-S on $15 \mu \mathrm{m} \mathrm{SiO}_{2} / \mathrm{Si}$ sample was mounted on a six-axis alignment stage at eucentric height and held in a chamber which was kept under high vacuum conditions $\left(<7 \times 10^{-6} \mathrm{mbar}\right) . \mathrm{Ga}^{+}$ions were generated, accelerated, and focused onto the sample by an ion gun situated in the same chamber. We set the machine to etch ring resonators and taper waveguide using an automated Stream file routine. The processing starts with etches defining 20 block patterns at an ion beam current of $150 \mathrm{pA}$. The depth of the ring resonators and taper waveguides was about $1 \mu \mathrm{m}$. A finer etching using a Pattern file then follows. The sample was tilted to $3.8^{\circ}$ and the magnification increased to $5000 \mathrm{X}$ before the final etch using beam currents below $350 \mathrm{pA}$. The last etch uses the smallest spot size and leads to smooth sidewalls. These steps allow us to etch smooth, vertical, high-resolution faces on the input and output waveguides as shown in Fig. 4.
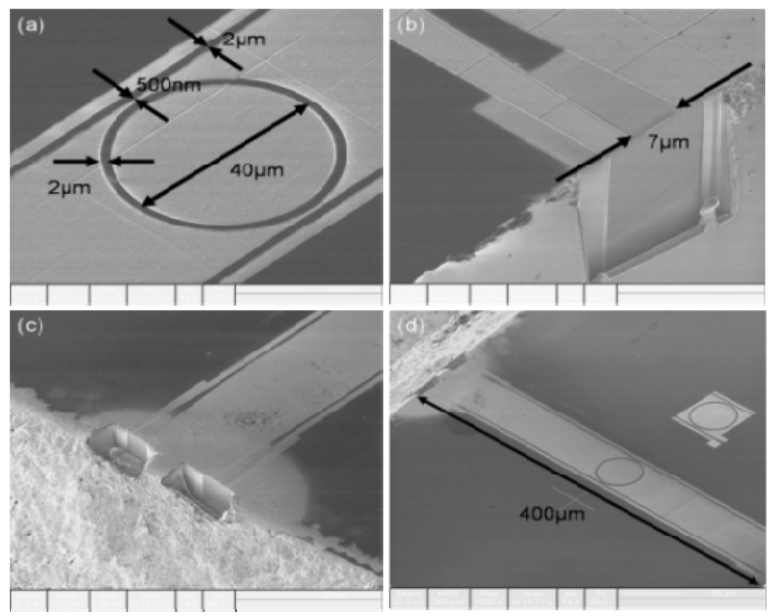

Fig. 4 FIB-etched ring resonator on CVD-grown Ge-Sb-S thin film.

\section{CONCLUSION}

Ge-Sb-S amorphous thin films have been successfully fabricated by CVD technique. The optical properties of these CVD-grown Ge-Sb-S amorphous thin films have been studied by UV-VIS-NIR spectroscopy with Swanepoel's method. The composition and surface morphology of these films have also been characterized by micro-Raman, SEM and EDX techniques. The FIB technique has been applied to fabricate $\mathrm{Ge}-\mathrm{Sb}-\mathrm{S}$ ring resonator. The characterization of the ring resonators are currently under investigation. The results of these CVD grown Ge-Sb-S thin films and FIB-etched ring resonator are very promising and have a great potential in optoelectronics, particularly as waveguides for optical integrated circuits applications.

\section{REFERENCES}

[1] K. Tanaka, Current Opinion in Solid State \& Materials Science, 1 (1996) 567.

[2] A. Zakery, S.R. Elliott, J. Non-Cryst. Solids, 330 (2003) 1-12.

[3] A. V. Kolobov, "Photo-Induced Metastability in Amorphous Semiconductors", Wiley-VCH, 2003.

[4] L. Koudelka, M. Frumar, M. Pisarcik, J. Non-Cryst. Solids, 41 (1980) 171-178.

[5] E. Marquez, T. Wagner, J.M. Gonzalez-Leal, A.M. Bernal-Oliva, R. Prieto-Alcon, R. Jimenez-Garay, P.J.S. Ewen, J. Non-Cryst. Solids, 274 (2000) 62-68.

[6] E. Marquez, A.M. Bernal-Oliva, J.M. Gonzalez-Leal, , R. Prieto-Alcon, T. Wagner, J. Phys. D: Appl. Phys. 39 (2006) 1793-1799.

[7] J. Hu, VTarasov, A. Agarwal, L. Kimerling, Optics Express, 15 (2007) 2307-2314.

[8] C.C. Huang, K. Kenton, D.W. Hewak, Optical Materials, 29 (2007) 1344-1347.

[9] V. E. Krohn, G.R. Ringo, Appl. Phys. Lett., 27 (1975) 479-481.

[10] D. Freeman, S. Madden, B. Luther-davies, Optics Express, 13 (2005) 3079-3086.

[11] R. Swanepoel, J. Phys. E: Sci. Instrum., 16 (1983) 1214-1222. 